\title{
Sequence Resource of Bacterial Communities Associated with Hemp in Ohio
}

\author{
Matthew Willman, ${ }^{1}$ Harold M. Keener, ${ }^{2}$ and Maria-Soledad Benitez ${ }^{1, \dagger}$ \\ ${ }^{1}$ Department of Plant Pathology, The Ohio State University, Wooster, OH 44691 \\ ${ }^{2}$ Department of Food, Agriculture and Biological Engineering, The Ohio State University, Wooster, \\ $\mathrm{OH} 44619$
}

\begin{abstract}
In spite of changes in regulation and increased hemp cultivation and commercialization, information on hemp best production practices in the United States is scarce. Due to hemp's human consumption, particularly as concentrated oil, hemp is often grown with low synthetic chemical inputs. Therefore, microbiome management and the use of microbial inoculants are important tools to increase hemp productivity. As a first step to characterize the hemp core microbiome, we completed a survey of bacterial communities associated with different tissue types of plants grown in a field in Ohio. This local dataset could be integrated with further hemp microbiome surveys to identify potential beneficial interactions relevant to plant health and productivity but also to evaluate microbiome impacts on product efficacy and safety.
\end{abstract}

Due to recent changes in regulation and to its potential of high economic revenues, there is increased interest from growers, industry, and researchers in industrial hemp. Industrial hemp refers to the cultivars of Cannabis sativa L. with a dry mass $\delta-9$ tetrahydrocannabinol concentration of $0.3 \%$ or less (USDA Agricultural Market Service 2019). Most popular hemp varieties are grown for cannabidiol production (CBD) (Hemp Benchmarks 2020; Mark et al. 2020). CBD-based supplements have gained interest due to potential applications in human health, with current evidence supporting CBD use for management of certain types of epilepsy (Abu-Sawwa et al. 2020). Depending on the variety, industrial hemp may have additional uses, including seed and other food supplements, fiber for textiles, and even construction (Clarke and Merlin 2016; Fike 2016).

Since its approval for production in certain U.S. states, academic research in hemp is focusing on development of best production practices. With CBD, the end product with greatest economic interest (Mark et al. 2020), being for direct human consumption, particularly as concentrated oil, the industry and its consumers are looking into practices with low synthetic chemical inputs and environmental impact (Madhusoodanan 2019). For example, from the 47 pesticide products currently registered for use in hemp (EPA 2020), 46 represent microbialbased or -derived products.

The successful application and performance of microbial-based products is dependent on our understanding of both management practices and resident microbiota in plants (Massart et al. 2015). Bacteria and other microorganisms colonize plant surfaces as epiphytes, as well as reside in inter- and intracellular spaces as endophytes (Kandel et al. 2017). Furthermore, each plant compartment selects for a distinct bacterial community, and bacterial community composition in hemp and other plant hosts is driven by soil characteristics, land management, and plant cultivar (Scott et al. 2018; Winston et al. 2014). Due to the regulated history of hemp and other Cannabis cultivars, a small number of studies have documented characteristics of its

${ }^{\dagger}$ Corresponding author: M.-S. Benitez; benitezponce.1@osu.edu

The author(s) declare no conflict of interest.

Accepted for publication 5 November 2020.

\author{
Keywords \\ agriculture, bacterial communities, \\ bacteriology, endophytes, \\ hemp, microbiome, Ohio, \\ rhizosphere and phyllosphere
}




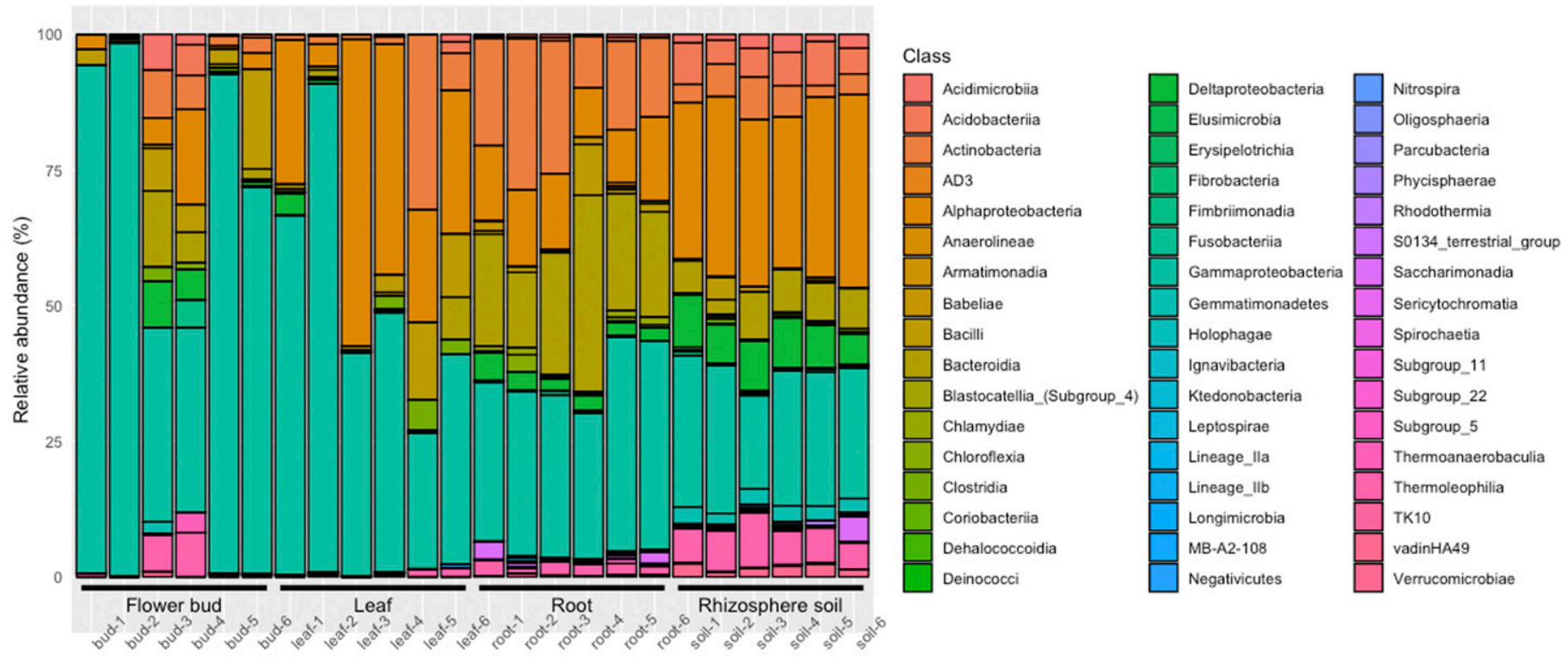

Sample

Fig. 1. Relative abundance of bacterial classes recovered from different tissue types of hemp grown in a field in Ohio. Tissue was sampled 2 months after transplant and surveyed for bacterial diversity using amplicon sequencing of the 16S ribosomal DNA V5-V7 region.

associated microbiota (Scott et al. 2018; Thompson et al. 2017). As hemp production becomes more widespread, opportunities for microbiome management, through augmentation or cultural practices will arise. To contribute to our knowledge and understanding of hemp microbial communities, we present an amplicon sequencing survey of bacterial communities associated with multiple tissue types in field grown hemp in Ohio.

\section{Materials and Methods}

This work was performed under approved University Hemp Cultivation and Research application through the Ohio Department of Agriculture. Hemp clones (C. sativa 'Tangerine') were transplanted into a field site at the Ohio State University (OSU) agricultural research station in Wooster, $\mathrm{OH}$ (latitude 40.7787 and longitude -81.9303, elevation $311 \mathrm{~m}$; Wooster silt loam) on 8 August 2019. Prior to planting, transplants were treated against insects and pests with a mixture of insecticides and fungicides. Plants were grown in raised beds fitted with black agricultural plastic mulch, with drip irrigation. Field conditions during the growing season, as recorded by the research station weather system (available through CFAES weather system; https://www.oardc.ohio-state.edu/weather1/) were $0.21 \mathrm{~cm}$ of precipitation, with average air temperature of $19.1^{\circ} \mathrm{C}\left(25.6\right.$ and $13.2^{\circ} \mathrm{C}$ average maximum and minimum, respectively), average relative humidity of 2.09 , and average soil temperature of $19.8^{\circ} \mathrm{C}$. Six plants were collected 2 months after planting. For each individual plant, rhizosphere soil, roots, leaves, and flower buds were collected. For rhizosphere soil, the sample was collected after shaking off loosely attached soil. The soil closest to the root was brushed off and stored frozen. Roots, leaves, and flower buds were combined per plant, washed by immersion in $70 \%$ ethanol followed by $10 \%$ bleach, and rinsed in sterile distilled water to minimize recovery of epiphytic microbiota. Tissue was blotted dry and stored frozen at $-80^{\circ} \mathrm{C}$ immediately after sampling. Total DNA was extracted from freeze-dried material using the Qiagen Plant DNA extraction minikit, following the manufacturer's recommendations. DNA quantity and purity were assessed through agarose gel electrophoresis run with GeneRuler $1 \mathrm{~kb}$, followed by image analysis through a custom protocol in ImageJ (Schneider et al. 2012). 16S ribosomal RNA amplicon libraries were prepared and sequenced at the Molecular, Cellular and Imaging Center at the OSU Wooster campus. A seminested PCR approach, modified from Beckers et al. (2016) and targeting $16 \mathrm{~S}$ ribosomal DNA variable regions $\mathrm{V} 5$ to $\mathrm{V} 7$ was used to maximize recovery of bacterial PCR products from total plant DNA extracts. For this, a first PCR was run for 15 cycles with the primers 799F (5'-AACMGGATTAGATACCCKG-3') and 1391R (5'-GACGGGC 
GGTGWGTRCA-3') (Chelius and Triplett 2001; Walker and Pace 2007). Then, a 1:20 dilution of PCR product was used as template for the second $P C R$ and run for 10 cycles with primer pair 967F (5'-CAACGCGAAGAACCTTACC-3') (Sogin et al. 2006) and 1391R (5'-GACGGG CGGTGWGTRCA-3') fused with Illumina MiSeq adapters. Individual sample indexes were then added through a final eight amplification cycles, following Illumina's dual-indexing approach. PCR products were then pooled in equimolar concentration, and the pooled library was size selected using Blue Pippin Prep (Sage Science) with a $2 \%$ agarose gel cassette and set to collect products of approximately $600 \mathrm{bp}$ to enrich for bacteria and exclude plant mitochondrial products (approximately 1,000 bp with Illumina adapter). Amplicon libraries were then pooled with other samples and analyzed by paired-end sequencing of 300 cycles on the MiSeq (Illumina, Inc.) platform (24 hemp samples represented 13\% of the run).

Sequence read quality was evaluated using fastqc v0.11.5 (Andrews 2010) and MultiQC v1.8 (Ewels et al. 2016). Primer and adapter were removed using cutadapt v2.5 (Martin 2011). Reads were imported to the DADA2 R package v1.14.0 (Callahan et al. 2016). DADA2 was then used to trim and filter reads, infer sequence variants, obtain paired-end amplicon sequence variants (ASVs), and assign taxonomy to ASVs. For taxonomy assignment, reads were compared against SILVA database version 132 (Quast et al. 2013). Sequence data were submitted to NCBl's Sequence Read Archive under BioProject number PRJNA641545.

Dataset description. In total, 1,940,575 reads were recovered for these 24 samples, with an average of 80,857 reads per sample. After filtering out ASVs assigned to plant mitochondria or chloroplast, other eukaryotes, or unknown, $99 \%$ of the recovered reads were of bacterial or archaeal origin. On average, a greater number of amplicon contigs (ac) and bacterial ASVs (bASVs) were recovered from rhizosphere soil (19,280 $\pm 12,658$ ac and 2170 bASVs) and leaf samples (28,663 $\pm 7,606$ ac and 342 bASVs) compared with buds $(16,869 \pm 6,360$ ac and 181 bASVs) and roots $(8,570 \pm 4,113$ ac and $1141 \mathrm{bASVs})$. Bacterial communities in leaves and buds were dominated by members of the classes Gammaproteobacteria and Alphaproteobacteria (Fig. 1), whereas bacterial communities on the roots were dominated by Gammaproteobacteria, followed by Bacteroidia and Actinobacteria. Alphaproteobacteria, Gammaproteobacteria, and Deltaproteobacteria dominated rhizosphere soil samples. This initial survey of the bacterial microbiome associated with the hemp cultivar Tangerine provides the first snapshot of microbial communities associated with this crop in Ohio. As more hemp is grown locally and regionally, this dataset will provide a baseline to evaluate microbiome variation across sites and hemp cultivars, as well as the presence of potential beneficial or pathogenic microorganisms. We aim to expand our studies of specific associations between variety, environment, agricultural management, and microbial communities, all relevant for understanding best production practices and product safety for the CBD market.

\section{Acknowledgments}

We thank L. Taylor for help with sampling and report review.

\section{Literature Cited}

Abu-Sawwa, R., Scutt, B., and Park, Y. 2020. Emerging use of Epidiolex (cannabidiol) in epilepsy. J. Pediatr. Pharmacol. Ther. 25:485-499.

Andrews, S. 2010. FastQC: A Quality Control Tool for High Throughput Sequence Data. http://www.bioinformatics.babraham.ac.uk/projects/fastqc/

Beckers, B., Op De Beeck, M., Thijs, S., Truyens, S., Weyens, N., Boerjan, W., and Vangronsveld, J. 2016. Performance of 16s rDNA primer pairs in the study of rhizosphere and endosphere bacterial microbiomes in metabarcoding studies. Front. Microbiol. 7:650.

Callahan, B. J., McMurdie, P. J., Rosen, M. J., Han, A. W., Johnson, A. J. A., and Holmes, S. P. 2016. DADA2: High-resolution sample inference from Illumina amplicon data. Nat. Methods 13:581-583.

Chelius, M., and Triplett, E. 2001. The diversity of archaea and bacteria in association with the roots of Zea mays L. Microb. Ecol. 41:252-263.

Clarke, R. C., and Merlin, M. D. 2016. Cannabis domestication, breeding history, present-day genetic diversity, and future prospects. Crit. Rev. Plant Sci. 35:293-327.

EPA. 2020. Pesticide Products Registered for Use on Hemp. U.S. Environmental Protection Agency. https://www.epa.gov/pesticide-registration/pesticide-productsregistered-use-hemp
Ewels, P., Magnusson, M., Lundin, S., and Käller, M. 2016. MultiQC: Summarize analysis results for multiple tools and samples in a single report. Bioinformatics 32:3047-3048.

Fike, J. 2016. Industrial hemp: Renewed opportunities for an ancient crop. Crit. Rev. Plant Sci. 35:406-424.

Hemp Benchmarks. 2020. August 2020 Production Survey Report. https://www. hempbenchmarks.com/

Kandel, S. L., Joubert, P. M., and Doty, S. L. 2017. Bacterial endophyte colonization and distribution within plants. Microorganisms 5:77.

Madhusoodanan, J. 2019. Can cannabis go green? Nature 572:S8-S9.

Mark, T., Shepherd, J., Olson, D., Snell, W., Proper, S., and Thronsbury, S. 2020. Economic Viability of Industrial Hemp in the United States: A Review of State Pilot Programs. Economic Information Bulletin EIB-217, United States Department of Agriculture, Economic Research Service. https://www.ers.usda.gov/webdocs/publications/95930/eib-217.pdf?v=5537.7

Martin, M. 2011. Cutadapt removes adapter sequences from high-throughput sequencing reads. EMBnet. J. 17:10-12.

Massart, S., Martinez-Medina, M., and Jijakli, M. H. 2015. Biological control in the microbiome era: Challenges and opportunities. Biol. Control 89:98-108. 
Quast, C., Pruesse, E., Yilmaz, P., Gerken, J., Schweer, T., Yarza, P., Peplies, J., and Glöckner, F. O. 2013. The SILVA ribosomal RNA gene database project: Improved data processing and web-based tools. Nucleic Acids Res. 41:D590-D596.

Schneider, C. A., Rasband, W. S., and Eliceiri, K. W. 2012. NIH Image to ImageJ: 25 Years of image analysis. Nat. Methods 9:671-675.

Scott, M., Rani, M., Samsatly, J., Charron, J.-B., and Jabaji, S. 2018. Endophytes of industrial hemp (Cannabis sativa L.) cultivars: Identification of culturable bacteria and fungi in leaves, petioles, and seeds. Can. J. Microbiol. 64:664-680.

Sogin, M. L., Morrison, H. G., Huber, J. A., Welch, D. M., Huse, S. M., Neal, P. R., Arrieta, J. M., and Herndl, G. J. 2006. Microbial diversity in the deep sea and the underexplored "rare biosphere.". Proc. Natl. Acad. Sci. U.S.A. 103:12115-12120.

Thompson, G. R., III, Tuscano, J. M., Dennis, M., Singapuri, A., Libertini, S., Gaudino, R., Torres, A., Delisle, J. M. P., Gillece, J. D., Schupp, J. M., and Engelthaler, D. M.
2017. A microbiome assessment of medical marijuana. Clin. Microbiol. Infect. 23 : 269-270.

USDA Agricultural Market Service. 2019. Establishment of a domestic hemp production program. Doc. No. AMS-SC-19-0042; SC19-990-2IR. Federal Register 84 No. 211. https://www.govinfo.gov/content/pkg/FR-2019-10-31/pdf/ 2019-23749.pdf

Walker, J. J., and Pace, N. R. 2007. Phylogenetic composition of Rocky Mountain endolithic microbial ecosystems. Appl. Environ. Microbiol. 73: 3497-3504.

Winston, M. E., Hampton-Marcell, J., Zarraonaindia, I., Owens, S. M., Moreau, C. S., Gilbert, J. A., Hartsel, J., Kennedy, S. J., and Gibbons, S. M. 2014. Understanding cultivar-specificity and soil determinants of the Cannabis microbiome. PLoS One 9: e107415. 